BNL 37621

INFORMAL REPORT
BNL -37621

vE86 006440

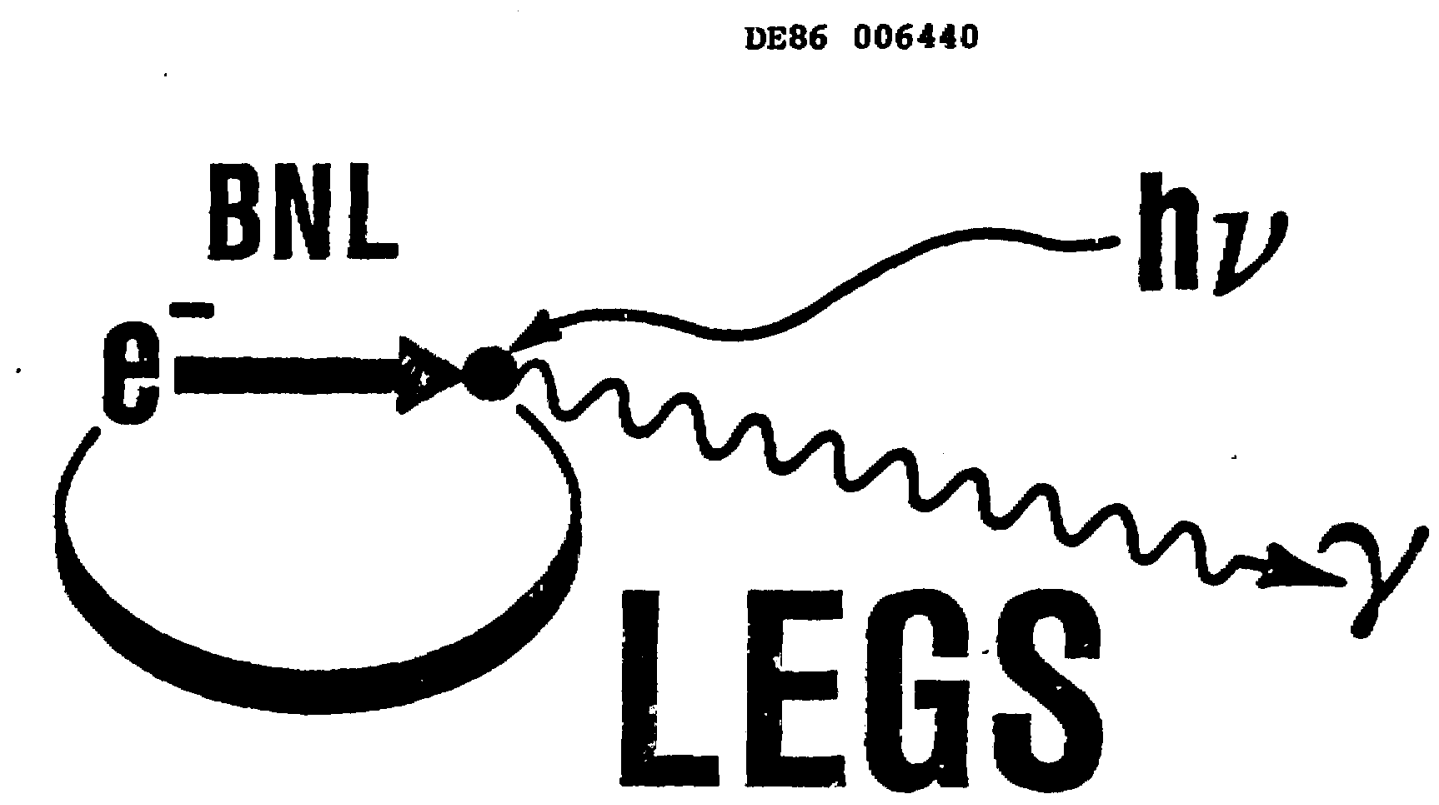

PHYSICS WITH POLARIZED PHOTONS

G. Matone

Physlcs Department

Brookhaven National Laboratory

Upton, New York 11973

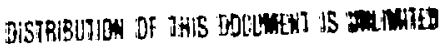

October 85

BROOKHAVEN NATIONAL LABORATORY

ASSOCIATE UNIVERSITIES, INC.

LNOER CONTRACT NO. DE-ACO2-76CHOCOHG WITH THE

UNITED STATES DEPARTMENT OF ENERGY 
ENL 37622

INIORMAL REPORI

\title{
PHYSICS WITH POLARIZED PHOTONS
}

\author{
G. Matone \\ Physics Department \\ Brookthaven National Laboratory \\ Upton, Yeu York 11973
}

October 85 
DISCLATMFR

Th1. report wa prepared as an account of work sponsored by an apency of the United States Government. Netther the United Staten Government nor any arency thereof, nor any of thetr employees, nor any of thetr emiractors, suhcontraccors, or their employees, makes any warranty, express or Impiled, or assumes any legal liablitty or responstbility for the acsurincy, completeress, or usefulness of any information, apparatus, prodist, or process diaclosed, or represents that its use would not infringe privately ownes rights. Reference hereln to any opectflc commerclal product, process, or service by trade name, trademark, manufacturer, or otherwlse. does not necessarily constitute or Imply its endoracment, recommendation, or favoring by the United Stetea Government or any agency, contractor or subcontractor thereof. The views and opinions of authors expressed heretn do no: necessarfly state or reflect those of the United States Covernment or any agency, contractor or mubcontractor thereof. 


\section{PHOTON POLARIZATION}

The availability of fully polarized and nonochroatic photon beano obtained with Compton scattering at Frascati ${ }^{(1)}$ and Brookhaven (2) has stimilated over the past few years considerable interest in the role that polarizarion can have in the interaction of real and virtual photone with nuelear anter. (3) In these two cases the structured of the interaction are very aimilar
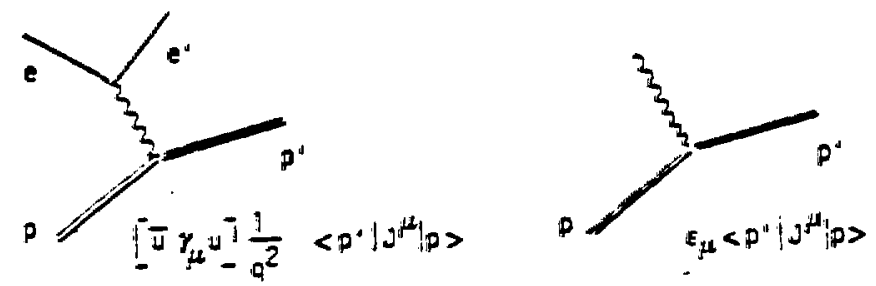

and both lead to a factorized form for the differential cross section

$$
d o \sim I_{\mu v} L^{\mu \nu}
$$

shere

$$
\begin{aligned}
& \left.I^{\mu \nu} \sim \mathrm{p}^{\prime}\left|\mathrm{J}^{\mu}\right| \mathrm{p}>\mathrm{p}^{\|}\left|\mathrm{J}^{\nu}\right| \mathrm{p}\right\rangle^{*}
\end{aligned}
$$

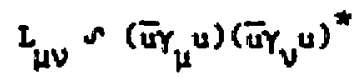

$$
\begin{aligned}
& L_{\mu \nu} \sim \Sigma_{\lambda \lambda}, \varepsilon_{\mu}^{\lambda} \rho \rho_{\lambda \lambda}, \varepsilon_{v}^{\lambda^{* *}}
\end{aligned}
$$$$
\text { for virtual photons }
$$

and $\varepsilon_{\mu}^{\lambda}$ is the photon polarization vector.

The polarization density atrix $\rho_{\lambda \lambda}{ }^{\prime}$ is usully defined for real photone, but woving in the Breit frame defined at the frane in which the electron ocetcers backward with no loss in energy, the anelogous quanticy can be defined also for virtuel photone. (4) In a very elegant way this allows for a complete analogy between the two cases and furniahes a powerful nethod to define the polerizetion atate lleo for virtual photons.

The photon polarization é entera in the definition of the vector potential of the radiation 


$$
\bar{\lambda}=\bar{c} e^{i(\bar{k} \cdot \bar{r}-k t)}
$$

and is in general a complex vector $e^{-\cdot} e^{-*}=1$ that for real photors is perpendicular to the photon momentua $\bar{k}, \bar{e}^{\cdot} \bar{k}=0$. In an arbitrary coordiatce system vith unit vectors $\bar{\varepsilon}_{x}$ and $\bar{\varepsilon}_{y}$ auch that $\bar{E}_{x}, \bar{\varepsilon}_{y}$ and $\bar{k}$ form a right-handed orthogonal coordinate system, any pure atate of polarization $\vec{e}_{i}$ can be expreved by

$$
\bar{c}_{i}=\Sigma_{\lambda} c_{i \lambda} \bar{\varepsilon}_{\lambda} \quad(\lambda-x, y) .
$$

Any photon bean is a wixed state or an incoherent sin of pure states $\bar{e}_{i}$ each occuring with statistical weights $p_{i}$. If we define the unitary density werix

$$
P_{\lambda \lambda^{\prime}}=\Sigma_{i} p_{i} c_{i \lambda}^{\star} c_{i \lambda}
$$

the crose section ( 1 ) for the photon absorption by an hadronic systen can be wricten as

$$
d \sigma \sim \Sigma_{\lambda \lambda}, f^{\lambda} \rho_{\lambda \lambda}, f^{\star \lambda}=\operatorname{Tr}\left(f_{p f}^{*}\right)
$$

where the aplitudes

$$
\epsilon^{\lambda}=e_{\mu}^{\lambda}\left\langle p\left|J^{\mu}\right| p\right\rangle=e_{\mu}^{\lambda} T^{\mu}
$$

deacribe the photon interaction with the electromagnetic current $\mathrm{j}^{\prime \prime}$. The form of the cross section (7) holds whether the process is coapletely differencial or than sone of the particle nomenta or polarization are not observed. We can now specialize eqs. $(5),(6),(7)$ to the following different cases.

Linear Polarization. - Two orthogonal states of linen polarization can be written as: 


$$
\bar{e}-\cos \phi \bar{\varepsilon}_{x}+\sin \phi \bar{\varepsilon}_{y}, \bar{e}^{\prime}=-\sin \phi \bar{\varepsilon}_{x}+\cos \phi \bar{\varepsilon}_{y}
$$

where $\phi$ is an arbitrary angle between $\bar{e}$ and $\bar{e}_{x}$. If we define the ban polarization $P$ se such that

$$
p_{1}=\frac{1}{2}(1+P), p_{2}=\frac{1}{2}(1-P), p_{1}+p_{2}=1
$$

where $p_{1,2}$ are the probabilities to find a photon in the atates $\vec{e}, \bar{e} \cdot$, then the denoity matrix (6) becomes

$$
\rho_{\lambda \lambda^{\prime}}=\left\|\begin{array}{ll}
\frac{1}{2}(1+P \cos 2 \phi) & \frac{1}{2} P \sin \phi \\
\frac{1}{2} P \sin 2 \phi & \frac{1}{2}(1-P \cos 2 \phi)
\end{array}\right\|
$$

and the cross section (7) for the detection of one particle is:

$$
d \sigma \sim \frac{\left|T_{x}\right|^{2}+\left|T_{y}\right|^{2}}{2}+P \frac{\left|T_{x}\right|-\left|T_{y}\right|^{2}}{2} \cos 2 \phi+P \operatorname{Re}\left(T_{x} T_{y}^{*}\right) \sin 2 \phi \text {. }
$$

The (2申) dependence of eq. (12) reflects the symetry of the problen under the traneforation $\phi+\phi+3$.

In the anse of no-polarization $(p m 0)$, eq. (12) reproduces the unual result

$$
d \sigma \sim \frac{\left|T_{x}^{2}\right|+\left|T_{y}^{2}\right|}{2}
$$

for unpolerized photons.

Gircular Polarization. - For the case of circular polarizution the electric and angnetic field strengthe of the radiation, rotate with conatant aplitudel and the two states of right-handed and left-handed circular polarization are siven by 


$$
\vec{e}_{R, L}=\frac{1}{\sqrt{2}}\left(\vec{\varepsilon}_{x} \pm i \bar{\varepsilon}_{y}\right)
$$

The density matrix and the cross section becone respectively:

$$
\rho_{\lambda \lambda}=\left\|\begin{array}{ll}
1 / 2 & -i \quad P / 2 \\
i \quad P / 2 & 1 / 2
\end{array}\right\|
$$

and

$$
d \sim \frac{\left|T_{x}\right|^{2}+\left|T_{y}\right|^{2}}{2}+P \operatorname{Id}\left(T_{x} T_{y}^{*}\right)
$$

where $P$ is the degree of circular polarization of the bea defined as in (10). Elliptic Polarization. - Analogous to the case of linear polarization the vectors wy, for the case of elliptic polerization, be written as: (5)

$$
\begin{aligned}
& \bar{e}-\left(a \cos \phi-i \xi_{b \sin \phi}\right) \bar{E}_{x}+\left(a \sin \phi+i \xi_{b \cos } \phi\right) \bar{E}_{y} \\
& \bar{e}^{\prime}-\left(b \cos \phi+i \xi_{\text {asin } \phi}\right) \bar{\varepsilon}_{x}+\left(b \sin \phi-i \xi_{a \cos }\right) \bar{E}_{y}
\end{aligned}
$$

where the real, positive numbers a and b satiafy

$$
a^{2}+b^{2}=1
$$

and $\xi$ is \pm 1 for right-handed and left-handed elliptic polarization respectively. The ratio $a / b$ is the ratio of the axes of the polarization ellipse, so that $a / b=1$ for circular polarizations and $a / b$ is infinite or zero for linear polarization. Analogous to the previous cases the cross section becomes: 


$$
\begin{aligned}
& d \sigma \sim \frac{\left|T_{x}\right|^{2}+\left|T_{y}\right|^{2}}{2}+P\left\{\left[\frac{\left|T_{x}\right|^{2}-\left|T_{y}\right|^{2}}{2} \cos 2 \phi+\right.\right. \\
& \left.\left.+\operatorname{Re}\left(T_{x} T_{y}^{*}\right)_{\sin 2 \phi}\right]\right\}\left(a^{2}-b^{2}\right)+2 \xi a b \operatorname{Ia}\left(T_{x} T_{y}^{*}\right)
\end{aligned}
$$

where $P$ is the degree of elliptical polarization.

Virtul Photong. - With the same formalism one can show that the state of polarization for virtual photons produced by an unpolarized electron bean correspond to an equal incoherent mixture of two pure polarization states: (6)

$$
\begin{aligned}
& \bar{e}=\sqrt{1+\varepsilon}\left(\cos \phi \bar{\varepsilon} x+\sin \phi \bar{\varepsilon}_{y}\right)+\sqrt{2 \varepsilon} \bar{\varepsilon}_{z} \\
& \bar{e}^{\prime}=\sqrt{1-\varepsilon}\left(-\sin \phi \bar{\varepsilon}_{x}+\cos \phi \bar{\varepsilon}_{y}\right)
\end{aligned}
$$

where

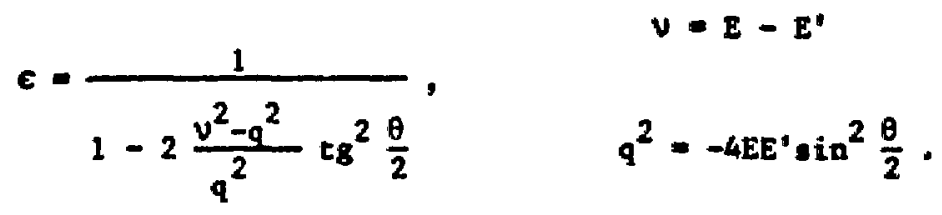

Dy conatructing the denaity antrix and accounting for gauge invariance one can deduce the very well known unpolarized lepton, unpolarized target and ane particle exclusive electroproduction cross section

$$
\begin{gathered}
d \sigma \sim \frac{\left|T_{x}\right|^{2}+\left|T_{y}\right|^{2}}{2}+\frac{\left|T_{x}\right|^{2}-\left|T_{y}\right|^{2}}{2} \cos 2 \phi+\frac{-q^{2}}{v^{2}}\left\|T_{z}\right\|^{2} \\
+\sqrt{\frac{-2 q^{2} \varepsilon(1+\varepsilon)}{v^{2}}} \text { Re }\left(T_{x}^{*} T_{z}\right) \cos \theta
\end{gathered}
$$

where the acimuthal angle is now referred to the electron reaction plane instead of the phocon polarization direction as it was for real photons. The terne in sins and sin2t vanish in (22) for exclusive reactions and the procens is 
described by only four structure functions. Generalizacion to exclusive n particle reactions with polarized electrons would lead to a total of 9 structure functions corresponding to the $3 \times 3$ independent terms of the bilinear products of the electonagnetic current. (7)

We noce that in (22) the ceras containing the longitudinal current (x subseripts) vanioh in the limit $q^{2}+0$ and reproduce the cross section for real and linearly polarized photon with poiarization

$$
E=\frac{2}{E^{2}+E^{\prime}}
$$

wich is kinewatics dependent. Ouing to paricy invariance the terms arising from $R \mathcal{C}\left(T_{x} T_{y}^{+}\right)$and $I_{m}\left(T_{x} T_{y}^{+}\right.$vanish in eqs. $(12),(16)$ and $(19)$ when no target polarization is involved and sumation over the unseen observables is carried out. On the other hand if particular final state configurations are selected these teras can be used either as polarization analysers or indicators of parity violacing effects. As indicated by eqs. (12), (13), (16) and (19), real photons can only probe the tranoverse part of the hadronic current whereas virtual photone enlarge the donain of investigation also to the longitudinal part. A longitudinal photon couples to a nuclear gystem in a different way then a crensverse photon does. Por instance, the pion exchange currents do not contribute to the first order to the longitudinal cross section which is re sensitive than the transverse croas section to the short range part of the nuclear wave function. In ather simplified description which however an contsin the essential physics involved one can say that the nucleus looks different for electront and photons. Electrons see the net electric charge-e-wheress photons see the individual charges of the nucleus constituents. (B) Consequently exchenge effects hardly influence the longitudinal component of the current but considerably af- 
fect the transverse couponents. This effect han been clearly denonstrated in the electrodisintegration of the ditteron at threshold performed at Minx. (9)

2. DEUTERON PHOTODISINTEGRATION WITH LINEARLY POLARIZED PHOTCNS

Following eq. (12), Partovi's approximation for the deuteriun disintegration in the $M$ is given by

$$
\frac{d \sigma}{d \Omega}=I_{0}(\theta)+P I_{1}(\theta) \cos 2 \phi
$$

where:

$$
\begin{aligned}
& I_{0}=a+b \sin ^{2} \theta+c \cos \theta+d \sin ^{2} \theta \cos \theta+c \sin ^{4} \theta \\
& I_{1}=f \sin ^{2} \theta+g \cos \theta \sin ^{2} \theta+h \sin ^{4} \theta .
\end{aligned}
$$

A measurement of the asymetry

$$
\Sigma(\theta)=\frac{I_{1}(B)}{I_{0}(\theta)}=\frac{\left|T_{x}\right|^{2}-\left|T_{y}\right|^{2}}{\left\|\left.T_{x}\right|^{2}+\mid T_{y}\right\|^{2}}
$$

at $\theta=\pi / 2$ in $C M$ and for $\left(10<E_{\gamma}<70\right)$ HeV was performed at Frascati a feu years ago with the Ladon monochromatic and polarized photon bean $\left(I_{Y} \sim 2 \times 10^{5},-1 ; s\right.$ 1). The reaults shown in Fig. 1, clearly indicated the need for an explicit inclusion of (REC) beyond the contribution given by the siegert theoren in the energy region $E_{Y}<100$ HeV where the unpolarized srose section $\left(\left|T_{x}\right|^{2}+\left|T_{y}\right|^{2}\right.$ is aloos completely insensitive to these effects.

In a ubsequent, more detsiled study of chis reaction $I_{0}(\theta)$ and $I_{1}(\theta)$ have been seperately messured a a function of enersy and the reaults normaized to the total sross section $\left(\sigma_{T}\right)$ sre reported in Fig. 2. 


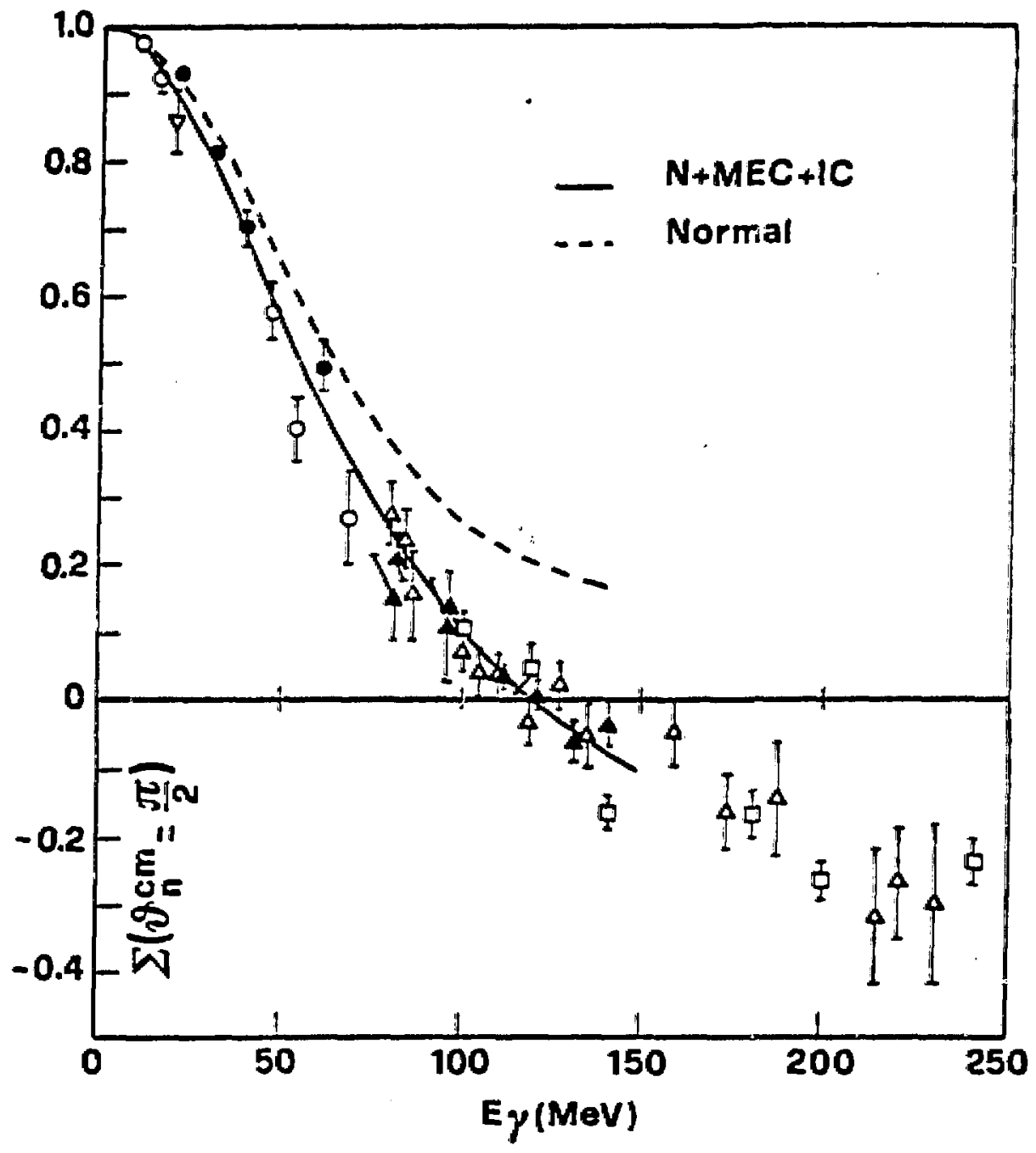

Fis. 1 Plot of the auymetry facter $\Sigma\left(\theta_{n}=\pi / 2\right)$ vs. laboratory gama-ray energy (MeV). Frascati data (solid circles) are compared with the results of earlier experiaents of Liu (solid and open triansles, Bef. 19), Del bianco et 1 . (inverted open triangle, Ref. 20), previous Frascati data (open circies, Ref. 21), Corbenko et al. (úpen squarea, Ref. 22). The theoretical curves have been obtained in Ref. 23 with the Rsc potential. The dashed line corresponds to the atandard Partovi appraxiantion (Ref. 24); the solid line reflects the inclusion of rec and IC. corrections. 

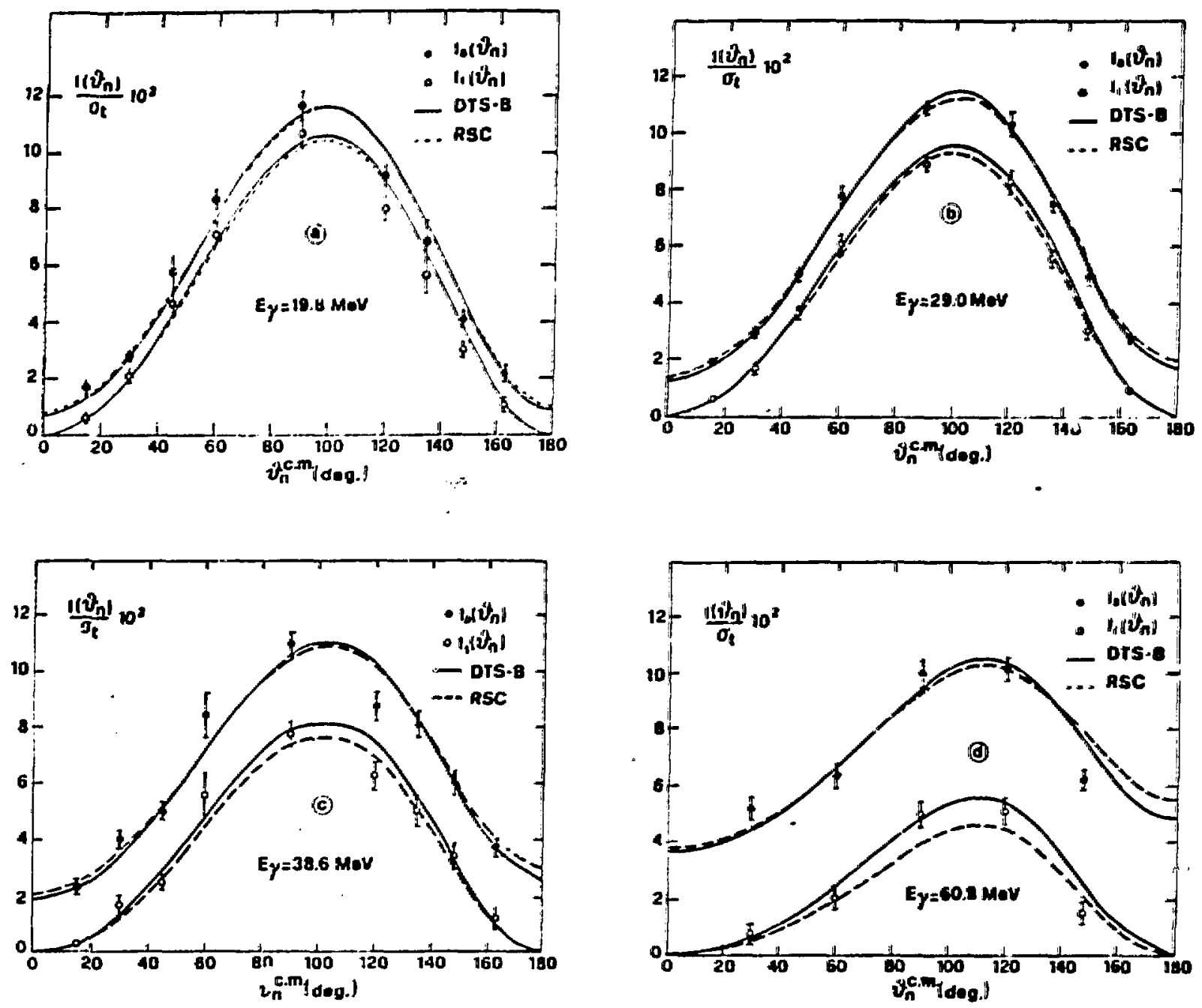

Pig. 2. Plote of $\left(I_{0}\left(\theta_{n}\right) / \sigma_{t}\right) 10^{2}$ and of $\left(I_{1}\left(\theta_{n}\right) / \sigma_{t}\right) 10^{2}$ as anction of the $a r$ neutron angles $\theta_{n}=(\pi-\theta)$ at: (a) E-19.8 MeV; (b) $29.0 \mathrm{MeV}$; (c) 38.6 MeV; (d) $60.8 \mathrm{MeV}$. Dazhed and solid lines repesent theoretical calculations of Reference 25 with the Reid soft-core (RSC) and the De Tourreil-Sprung (DTS-B) potentials, respectively. 
By assuming the values of $\sigma_{I}$ given by the fit of all the worldwide available data ${ }^{(10)}$ one can deduce the values for the forward differential cross section $I_{0}-(a+c)$ shown in Fig. 3 cogether with the Mainz, Louvain and Indiana results. The substantial agreement among all these experiments is renarkable.

Besides being extremely sensitive to the dynaic of the interaction, the term $\left\langle\left|T_{x}\right|^{2}-\left|T_{y}\right|\right) / 2$ in eq. (12) has the typical cos $2 \phi$-dependence that wakes it of invaluable help to observe saall ampitudes by interferences with the leading term. Typical example is the electric and agnetic aultipoles separation in the $\left(\gamma, \gamma^{\prime}\right)$-reaction on uuc!ei, as it has been pointed out on muny occasions. In particular the possibilicy to measure the $E 2 / M 1$ incerference term in the $\Delta-$ excitation appears very straight forward with linearly polarized photons.

The differential cross sections for meson photoproduction in $P_{3 / 2}$ state by a agnetic dipole or electric quadrupole absorption of a plane polarized photon are given by

$$
\begin{aligned}
& \mathrm{I}^{\mathrm{M} 1}(\theta, \phi) \sim \frac{1}{2} \sin ^{2} \theta(5-3 \cos 2 \phi)+\cos ^{2} \theta . \\
& I^{E 2}(\theta, \phi) \sim \frac{1}{2} \sin ^{2} \theta(1-\cos 2 \phi)+\cos ^{2} \theta
\end{aligned}
$$

respectively.

In the plane $\phi=0, I(M I) s$ const. but $I(E 2) s \cos ^{2} \theta$ and consequently a possible E2/ML interference term can be evidenced vith good accuracy. Since it is very difficult to extract unabigously a value for the E2 anplitude at resonance, the present experimental value for the $\mathrm{E} 2 / \mathrm{Ml}$ ratio is $-0.012 \pm 0.013$. (11) On the other hand the presence of an admixture of quadrupole strength in the $\Delta$-excitation would imaediately suggest quark d-state components in the baryons wave function as a direct consequence of the hyperfine splitting due to the residusl forces of $Q C D .(12)$ This has been discussed by several suthors also 


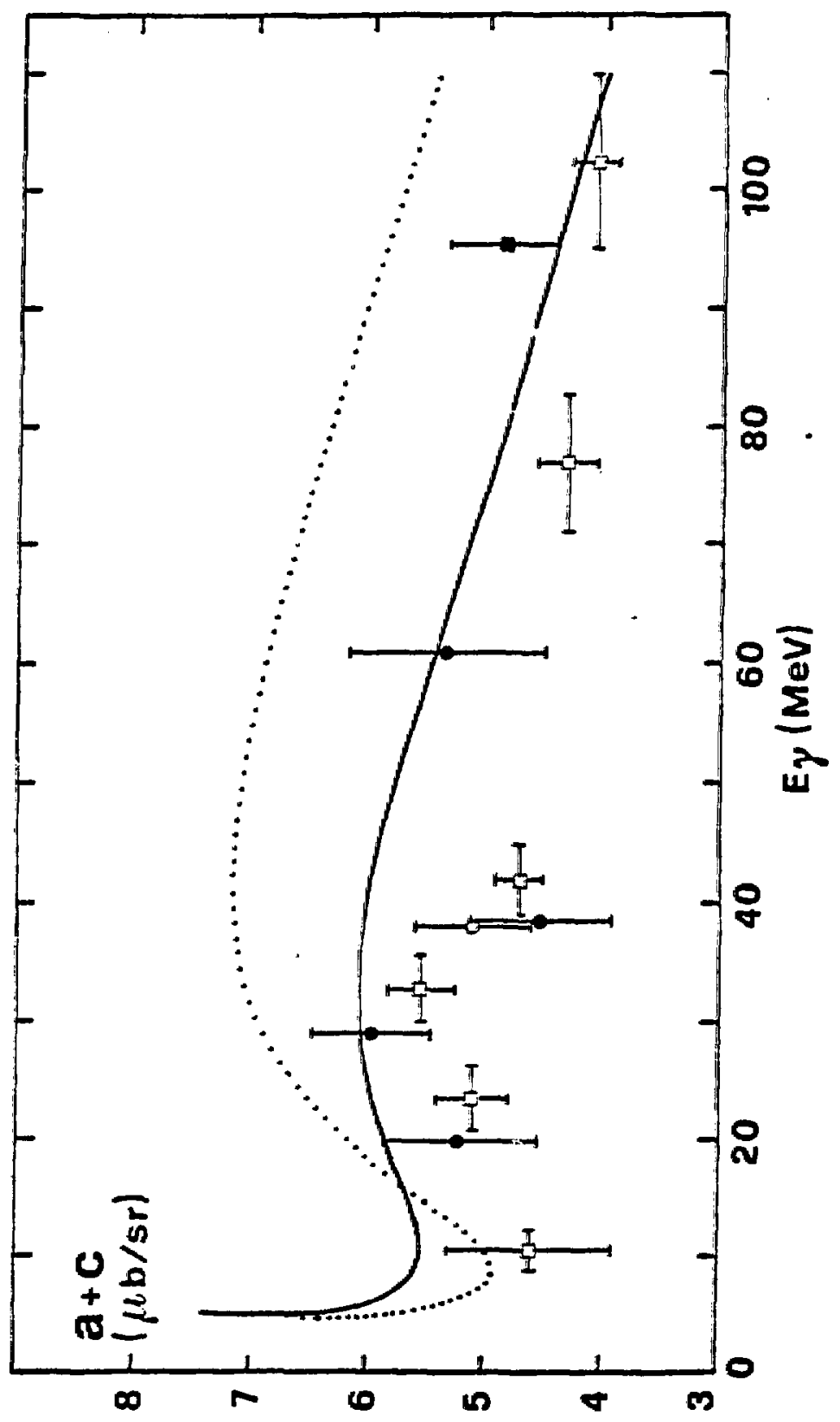

Fis. 3 Plot of our estizates (full circles), for the forward differeatial crose section (a+c), conpared with the experimental results of Ref. 26 (open squares), Ref. 27 (open circle) and Ref. 28 (full square). The theoretical calculations are due to Cabi et al. (Ref. 29) in IA (dotted line) and with relativitic corrections (full line) using the Paris potential. 
in connection with corrections to the static properties of the hadron, even if there are serious difficulties in the proper treatment of the center-of-aes motion of the nucleon bag. $(13,14)$

In a similar way, electric ( $(A)$ and magnetic (B) polarizabilities can be separated in the Compton scattering of plane polarized phocons on nucleone vhere eq. (12) specializes into

$$
d \sigma=\frac{d \sigma^{\prime \prime}+d \sigma^{\perp}}{2}+\frac{d \sigma^{\prime \prime}-d \sigma^{\perp}}{2} \cos 2 \phi
$$

where

$$
\begin{aligned}
& d \sigma^{\perp}=d \sigma_{0}^{\perp}-2\left(\frac{e 2}{M}\right)^{2}\left(\frac{E}{M}\right)^{2}(\alpha+\beta \cos \theta) \\
& d \sigma^{\prime \prime}=d \sigma_{0}^{\prime \prime}-2\left(\frac{e^{2}}{Y}\right)\left(\frac{E}{Y}\right)^{2}(B+\alpha \cos \theta) \cos \theta
\end{aligned}
$$

and $d \sigma_{0}$ is the no-structure nucleon cross section.

As it has been discussed extensively elsewhere, (15) these two quantities can be related to the usual expression for the electric and agnetic dipole wowents of a composite system

$$
\begin{array}{ll}
\alpha \sim\left\langle p\left|\bar{d}^{2}\right| p\right\rangle & \beta \sim\left\langle p\left|\bar{p}^{2}\right| p\right\rangle \\
\bar{d}=e \sum_{i} \hat{e}_{i} \bar{\tau}_{i} & \bar{E}=\mu_{p} \Sigma_{i} \hat{\alpha}_{i} \bar{\sigma}_{i}
\end{array}
$$

where $\hat{e}_{i}$ and $\hat{a}_{i}$ characterize the nature of the constituent quarks. From eqe. (32) it is evident how $\alpha, B$ are essentially related to the dimension of the nucleon bag and its internal structure respectively. Therefore they ay also depend on whether the nucleon is free or eabedded inside the nuclear anter and from this point of view a hydrogen-deuterium comparison could be highly illumating. Linearly polarized photons are the best tool to definitely masure these two very basic and largely unknown properties of the nucleons. 


\section{CIRCULARLY POLARIZED PHOTONS}

Circularly polarized photong appear to be very appealing when used together with polarized target.

The differential Compton eross section of eircularly polarized $\gamma$-raye on polarized electrons at rest is given by: (16)

$$
\begin{aligned}
\frac{\partial \sigma^{ \pm}}{d \Omega} & -\frac{1}{2} r_{0}^{2}\left(\frac{\omega_{2}}{\omega_{1}}\right)^{2}\left\{\left[\frac{\omega_{1}}{\omega_{1}}+\frac{\omega_{2}}{\omega_{1}}-\operatorname{in}^{2} \theta\right] \pm\left[\left(\frac{\omega_{2}}{\omega_{1}}-\frac{\omega_{1}}{\omega_{2}}\right) \cos \theta \cos \psi\right.\right. \\
& \left.\left.+\left(\frac{\omega_{2}}{\omega_{1}}-1\right) \sin \theta \sin \phi \sin \psi\right]\right\}=\Lambda(\theta) \pm B(\theta, \phi)
\end{aligned}
$$

where $r_{0}=e^{2} /\left(m c^{2}\right)$ and $\psi$ is the polar angle of the electron spin direction with respect to the incoming photon monetun. The energies of the incident and acattered photons $w_{1,2}$ are related by

$$
\omega_{2}=\frac{w_{1}}{1+\frac{\omega_{1}}{M}(1-\cos \theta)}
$$

and the upper-lower signs refer to an incident left-right circularly polarized photon. The first term in the equation gives the ordinary compton scatering cross section and the second gives the spin dependent one. This equation can be easily generalized for any electrons and it has been used for studying spin densities in ferro-magnetic materials $(\psi=0)(17)$. In a similar way the compton scattering on transverse $(\psi=\pi / 2)$ polarized electrons is currencly used co monitor the transverse beat polarization in storage rings. In both cases the relevant paraneter is always the aymetry

$$
\Sigma=\frac{d \sigma t-d \sigma t}{d \sigma^{t}+d \sigma^{t}}=\frac{B}{A} \frac{N^{t}-x t}{N^{t}+N^{\frac{1}{t}}}
$$


that measures the spin distributions $\mathrm{N}^{\dagger}$, $\mathrm{x}^{\dagger}$ inside the ensemble of electrons explored.

There is no resson, in principle, why this argunent could not be applicable to other systenn of fermions like nueleons in the aucleus or quarks or partons in a nucleon. It the case of an orienced qucleus this is jut the extension of the Compton scattering on bound nucleons there, besides isolating the spin depandent part of eq. (33), the esphesis is concentrated on the nucleon opin distribution. On the other hand, the electrons in the storage ring bunch are separated by relatively large distances and interact only electromagnetically, whereas nucleone or quarks are in close proximity and interact strongly: final state interactions could greatly complicate this potentially simple picture. Therefore in order to proceed slong this line we will ake the quite strong asumption that, during the sattering, one can neglect the interactione between the constituents. Moreover the wowentus transfer has to be high enough to resolve detaily on a length scale much less than the nucleus size, typically

$$
q^{2} \times 4 \omega_{1}(t) 2 \sin ^{2} \frac{\theta}{2} \geq 0.1 \mathrm{Gev}^{2}
$$

besides when $q^{2} \geq 0.7 \mathrm{GeV}^{2}$ the nucleon structure starts entering the gave and from the nucleon spin physics we paes to quark/parton spin physics. In this case the cross section should be exactly the electron-point like cross section (33) and eq. (35) becomes

$$
\Sigma=\frac{B}{A} \frac{\Sigma_{i} e_{i}^{2}\left(q_{i}^{+}-q_{i}^{\dagger}\right)}{\Sigma_{i} e^{2}\left(q_{i}^{\dagger}+q_{i}^{\dagger}\right)}
$$


where $e_{i}$ are the charges of the $u_{1} d$ quarks and $q^{+}, q^{+}$the probabilities to find thea with spins parallel (antiparallel) to the polarized proton. If there is negligible $L$ in the system then two quarka will have $S_{z}-1 / 2$ and one $S_{z}=-1 / 2$.

Bence the querk opine will be doninatly alligaed along the direction of the proton spin and so we expect $\Sigma>0$. If we take the su(6) wave functions for the proton, then the probabilities are ${ }^{(18)}$

$$
u^{4}=\frac{5}{9}, \quad u^{+}=\frac{1}{9}, \quad d^{+}-\frac{1}{9}, \quad d^{+}=\frac{2}{9}
$$

and we can quantify $\Sigma$ as follows:

$$
\Sigma^{Y P}=\frac{B}{A} \frac{\frac{4}{9}\left(\frac{5}{9}-\frac{1}{9}\right)+\frac{1}{9}\left(\frac{1}{9}-\frac{2}{9}\right)}{\frac{4}{9}\left(\frac{5}{9}+\frac{1}{9}\right)+\frac{1}{9}\left(\frac{1}{9}+\frac{2}{9}\right)}=\frac{5}{9} \frac{B}{A}
$$

Similarly for the "utron one fiad $\varepsilon^{\gamma n}-0$. From the experiaental point of vies, the size of the asymetry $(B / A)$ one has to look at, can be roughly evaluated by sseuming eq. (33) to be valid also for protons supposed to be point-like. Values of $B / A$ for a polarized proton at different photon energies are given in Fig. 4(a,b): at Eq. $\sim 700 \mathrm{MeV}$ and $\psi=0$ (longitudinally polarized protons), the aswetry $B / A$ at $\theta-\pi$ is $s 70 \%$ and hence $\Sigma \simeq 40 \%$. In these conditions, the wonentue transfer cones to be $q^{2} \times 8 \mathrm{CeV}^{2}$ and this should probably be wufficient to start seeing quark structure in the proton even though the unpolarized crose section from eq. (33) comes out to be $55 \cdot 10^{-33} \mathrm{cn}^{2} / \mathrm{sr}$. These conclusions appear to be rewarkable even if they have been obtained under very aimplifying conditions. Moreover, if they are really significant as they appear, their $q^{2}$-dependence could give important indications of the spin-distribution at the different levels of complexity of the hadronic anter: nuclei, nucleons, quarks. 


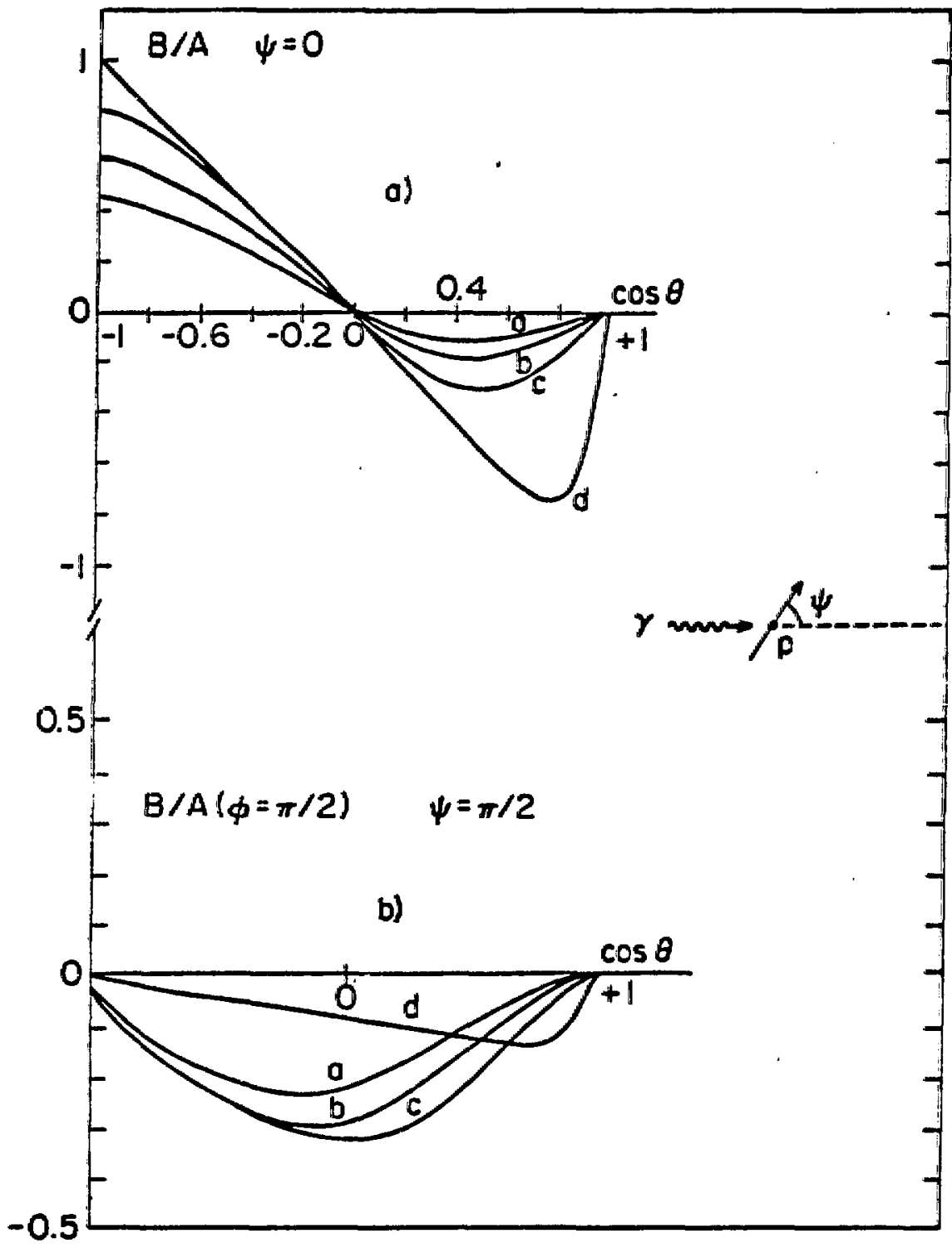

Fig. 4 Aymetry values at different energies for $\psi=0$ and $\psi-\pi / 2 ; \phi / 2$. Curves a), b), c), d) correspond to $\mathrm{E}_{\gamma}=300,500,1000,10,000 \mathrm{xeV}$
respectively. 
Within the linits of its validity, this argument could be pushed further on if, rather than scatter the photon, we consider absorbing it. In this case and under the aume kinematical conditions, photoabsorption by a quark in a polarized nucleon requires necessarily a spin flip:

$$
\begin{aligned}
& \gamma^{+}+q^{+}+q^{+} \\
& \gamma^{+}+q^{+}+q^{t}
\end{aligned}
$$

but $\gamma^{+} q^{\dagger}$ and $\gamma^{\dagger} q^{\dagger}$ cannot lead to absorption, only scatcering. Therefore:

$$
\begin{aligned}
& \sigma_{3 / 2} \sim \gamma^{\dagger} P^{\dagger} \sim \Sigma_{i} e_{i}^{2} q^{\dagger}
\end{aligned}
$$

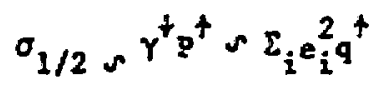

and once again one has

$I(x)-\frac{\sigma_{1 / 2}-\sigma_{3 / 2}}{\sigma_{1 / 2}+\sigma_{3 / 2}}-\frac{e_{u}\left(q_{d}^{\dagger}(x)-q_{u}^{\dagger}(x)+e_{d}^{2}\left(q_{d}^{\dagger}(x)-q_{d}^{\dagger}(x)\right)\right.}{e_{u}^{2}\left(q_{u}^{\dagger}(x)+q_{u}^{\dagger}(x)+e_{d}^{2}\left(q_{d}^{\dagger}(x)+q_{d}^{\dagger}(x)\right)\right.}$

where $x-q^{2} / 2 m v$ and $q_{u}^{\prime \prime} d$ are the quark $x$-distribution functions. For $x \geq$ .3 this ratio approaches the usual volume $5 / 9$ and 0 for proton and neutron respectively; but in the linit of real photons $(x+0)$, the unpolarized (qq) should dominate over the valence quarka forcing $\Sigma+0$ independently upon the valence quark spin distribtuion inside the target nucleons. Tests of eq. (40) have been perfomed already at SLAC in polarized electroproduction in the scaling region and the predictions of the simple quark nodel appear to be in fairly good agreenent with the data. (30)

Taking these concluoions literaly, quark opin distributions in polarized nucleons should be better studied by satcering real circular photons rather than aboorbing them on oriented quarike in polarized aucleons. 
Frow this point of view, circular photons could open new perspectives in the experimental nuclear physics scudies. 


\section{REFERENCES}

(1) L. Frederici et al., Nuovo Cimento 598 (1980) 247.

(2) A.M. Sandorfi et al., IEEE Trans. NS-30 (1983); LEGS Progrese Report BNL34938 ( 1984$)$.

(3) Proceedings of the Workshop on Intermediate Energy Nuclear Physics with Monochromatic and Polarized Photons, Frascai 1980, eds. G. Matone and S. Stipeich (Laboratori Nazionali di Erascati, 1980).

(4) A.J.G. Hey, Polarization effects in electron proton scattering, Report DL/R33 (1974).

(5) J. Motz et al., Rev. Mod. Phys. 41 (1969) 581.

(6) C.พ. Akerlof et al., Phys. Rev. 163 (1967) 1482.

(7) T.W. Donne1ly, 3rd Workshop of the Bates Users Theory Group (MIT, 1984 ).

(8) B. Ziegler, in (3), PP. 67-87.

(9) G.G. SImon et al., Nuclear Phys. A324 (1979) 277.

(10) M.P. De Pascale et al., Phys. Letters 119B (1982) 30.

(11) G. Kölbemann et al., Phys. Rev. D28 (1383) 28.

(12) D. Drechsel, Coincidence program planned of Mainz, Report MKPH-T/83-2 (1983).

(13) v. Vento et a1., Phys. Letters 1023 (1981) 97.

(14) I. Hulthage et 21., Nuclear Phys. A364 (1981) 322.

(15) G. Dattoli et al, Lett. Nuovo Cimento 19 (1977) 601.

(16) P.M. Platzmann et al.. Phys. Rev. 02 (1970) 3556.

(17) N. Sakai et al., Phys. Rev. Letters 37 (1975) 351.

(18) F.E. Close, An Introduction to Quarks and Partons (Academic Press, 1979).

(19) R.F. Liu, Phys. Rev. 1388 (1965) 1443.

(20) 1. Del Bianco et al., Nuclear Phys. 1343 (1980) 121.

(21) H. Del Bianco et 2!., Phys. Rev. Letters 47 (1981) 1118.

(22) W.G. Gorbenko et 2., Nuclear Phys. A381 (1982) 330.

(23) H. Arenhövel, Nuclear Phys. A374 (1982) 521c. 
(24) F. Partovi, Ann. Phys. 27 (1964) 79.

(25) A. Cambi et al., Phys. Rev. C26 (1982) 2358.

(26) R.J. Hughes at al., Nuclear Ph;9. A276 (1967) 329. A. Zieger ec al., Abstract of "11 th Europhysics Divisional Conference-Nuclear Physics vith Electronagnetic Probes", Paris, July 1-5, 1985 ed. by E.P.S. C17, 140 (1985).

(27) A. Ninane te 1., Can. J. Phys. 62 (19.4) 1104.

(28) H.0. Heyer et al., Phys. Rev. Lettery 52 (1984) 1759.

(29) A. Cambi et al., J. Phys. C: Nuclear Phys. 10 (1984) LIl, and ref. therein.

(30) Alquard, M.J. et al., Phys, Rey. Lett. 37 (1976) 1261; G. Baum et al., Phys. Rev. Lett. 51 (1983) 1135. 